\title{
Comparative proteomic analysis of atrial appendages from rheumatic heart disease patients with sinus rhythm and atrial fibrillation
}

\author{
WEN-JUN HUANG ${ }^{1,3^{*}}$, RUI ZHOU ${ }^{1 *}$, XIAO-RONG ZENG ${ }^{3}$, XIAO-QIU TAN ${ }^{3}$, ZHI-HUI CHENG $^{1}$, \\ MING-HAI TANG ${ }^{1}$, LAN-TU GOU ${ }^{1}$, LI-JUAN CHEN ${ }^{1}$, AI-PING TONG ${ }^{1}$, YONG HE$^{2}$ and JIN-LIANG YANG ${ }^{1}$ \\ ${ }^{1}$ State Key Laboratory of Biotherapy and Cancer Center, West China Hospital, West China Medical School, Sichuan University, \\ Chengdu 610041; ${ }^{2}$ Department of Cardiology, State Key Laboratory of Biotherapy, West China Hospital, \\ West China Medical School, Sichuan University, Chengdu 610041; ${ }^{3}$ Department of Electrophysiology, \\ Institute of Cardiovasology, Luzhou Medical College, Sichuan 646000, P.R. China
}

Received December 2, 2010; Accepted March 22, 2011

DOI: $10.3892 / \mathrm{mmr} .2011 .468$

\begin{abstract}
Atrial fibrillation (AF) is the most common form of arrhythmia encountered in clinical practice, and contributes to cardiovascular morbidity and mortality. Despite significant advances in the understanding of the mechanisms associated with AF, the number of effective biomarkers and viable therapeutic targets remains relatively limited. In this study, 2-DE and MS/MS analysis was used to identify differentially expressed proteins in human atrial appendage tissues from patients with $\mathrm{AF}(\mathrm{n}=4)$ compared to controls with sinus rhythm (SR; $n=5)$. All subjects had rheumatic heart disease. Following 2-DE analysis, Coomassie Brilliant Blue staining and MS/MS identification, a total of 19 protein spots were found to be differentially expressed between the AF and SR groups. By cluster and metabolic/signaling pathway analysis, these proteins were divided into three major groups: proteins involved in the cytoskeleton and myofilament, energy metabolism associated proteins, and proteins associated with oxidative stress. The proteins identified in this study may enable a better understanding of the molecular
\end{abstract}

Correspondence to: Dr Ai-Ping Tong, State Key Laboratory of Biotherapy and Cancer Center, West China Hospital, West China Medical School, Sichuan University, Chengdu 610041, P.R. China E-mail: aipingtong@gmail.com

Dr Yong He, Department of Cardiology, State Key Laboratory of Biotherapy, West China Hospital, West China Medical School, Sichuan University, Chengdu 610041, P.R. China

E-mail: zznnyeah@163.com

*Contributed equally

Abbreviations: RHD, rheumatic heart disease; AF, atrial fibrillation; SR, sinus rhythm; NYHA, New York Heart Association classification; 2-DE, 2-dimensional gel electrophoresis; HCM, hypertrophic cardiomyopathy

Key words: proteomics, rheumatic heart disease, atrial fibrillation, cytoskeleton, energy metabolism, oxidative stress mechanisms of AF, and may provide useful biomarkers and novel targets for drug development.

\section{Introduction}

Atrial fibrillation (AF) is the most common form of arrhythmia encountered in clinical practice, and contributes to cardiovascular morbidity and mortality $(1,2)$. The prevalence of $\mathrm{AF}$ has been shown to increase with age: in individuals 55-59 years of age, it has a prevalence of 0.7 , while in individuals 85 years of age or older, the prevalence increases to $17.8 \%(3,4)$. Consequently, though the number of patients with AF in the US was 2.3 million in 2001 (5), it has been estimated that this will reach 15.9 million by 2050 (6).

Effective therapy and prevention is crucial for the control of AF-related morbidity and mortality. However, the availability of effective biomarkers and viable therapeutic targets remains limited, despite recent significant advances in the understanding of the mechanisms associated with AF. Therefore, new methods leading to further insights into the underlying mechanisms of AF are required to identify effective biomarkers and novel therapeutic targets.

In recent years, proteomics has been used to identify diagnostic biomarkers for the early detection of cardiovascular disease, as well as novel therapeutic targets for its treatment (7). Using the proteomics method, the molecular mechanisms that contribute to AF were examined in several animal models of pacing-induced AF $(8,9)$. More recently, three different groups carried out a proteomic analysis of atrial fibrillation using human atrial appendage tissues (10-12). Combining metabolomic and proteomic analysis of human atrial fibrillation, Mayr et al (10) showed that the disrupted regulation of energy metabolites preceded the onset of postoperative AF. Modrego et al (11) performed a proteomic analysis to compare the expression of proteins between left atrial appendages (LAA) and right atrial appendages (RAA) obtained from patients with mitral valve disease with either sinus rhythm or permanent atrial fibrillation. Moreover, García et al (12) found that the down-regulation of the structural protein fibulin-1 
in atrial tissue from $\mathrm{AF}$ patients may reflect the myocardial structural changes that take place in patients with arrhythmia. These proteomic analyses of human atrial fibrillation have contributed to the understanding of the mechanisms leading to atrial fibrillation. However, all three studies investigated European populations; $\mathrm{AF}$ in Asian populations has yet to be investigated. Since the incidence of AF is related to race (5) and differs between developed and developing countries, we performed a proteomic analysis in a Chinese population to identify differentially expressed proteins in the human atrial appendage tissues of patients with $\mathrm{AF}(\mathrm{n}=4)$ compared to controls with sinus rhythm (SR; $n=5)$. All the subjects had with rheumatic heart disease, which is a significant cause of AF.

\section{Patients and methods}

Patients. The study comprised 9 subjects, all diagnosed with rheumatic heart disease (RHD). Four of the patients had previously been diagnosed with AF, while the other 5 had SR and served as controls. The clinical characteristics of the patients are summarized in Table I. The AF and SR groups were matched for the most relevant clinic variables. The diagnosis of AF was reached by evaluating medical records and 12-lead electrocardiogram (ECG) findings. Patients with AF had had the documented arrhythmia for at least 6 months. Controls with SR had never been on any antiarrhythmic drugs and were screened to ensure that they had never experienced AF by questioning regarding suggestive symptoms and analysis of 12-lead ECGs. Human right atrial appendage biopsies were obtained from the patients during standard cardiac surgery. Written informed consent was obtained from all patients, and the study procedures adhered to the guidelines of the Ethics Committee of the Affiliated Hospital of Luzhou Medical College and with the Helsinki Declaration of 1975, as revised in 2008. The samples were snap frozen in liquid nitrogen and stored at $-80^{\circ} \mathrm{C}$ until processing.

2-Dimensional gel electrophoresis (2-DE) and image analysis. 2-DE was was conducted as previously described (13) with minor modifications. Briefly, right atrial appendages were homogenized and sonicated in lysis buffer (7 M urea, $2 \mathrm{M}$ thiourea, 4\% CHAPS, 100 mM DTT, $0.2 \%$ ampholyte $\mathrm{pH} 3-10$; Bio-Rad, USA) containing protease inhibitor cocktail. IPG strips (17 cm, pH 7-10, non-linear; Bio-Rad) were passively rehydrated ( $2 \mathrm{mg}$ protein loading amount) for 12-16 h. Four replicates of each sample were run. Statistical analysis was performed by applying the student's t-test. Spots that showed consistent and significant differences $(>2$-fold, $\mathrm{p}<0.05)$ were selected for mass spectrometry (MS) analysis.

In-gel digestion, MS/MS analysis and protein identification. In-gel digestion of protein spots was performed using MS-grade Trypsin Gold (Promega, Madison, WI) according to the manufacturer's instructions. ESI-Q-TOF MS/MS analysis and protein identification were performed as described previously (14) with minor modifications. Briefly, mass spectra were acquired using a Q-TOF mass spectrometer (Micromass, Manchester, UK) coupled with an ESI ion source (Micromass). For MASCOT analysis, peptide and fragment mass tolerance were set at 0.1 and 0.2 Da respectively.
Table I. Clinical characteristics of the SR and AF patients.

\begin{tabular}{lcc}
\hline & $\mathrm{SR}(\mathrm{n}=5)$ & $\mathrm{AF}(\mathrm{n}=4)$ \\
\hline Age (years) & $47.4 \pm 3.6$ & $45 \pm 4.4$ \\
Gender (male/female) & $2 / 3$ & $2 / 2$ \\
NYHA & II & II \\
RVOT (mm) & $26.8 \pm 4.36$ & $26.2 \pm 5.77$ \\
LAD (mm) & $38.4 \pm 5.40$ & $55.26 \pm 9.98^{\mathrm{a}}$ \\
LVDd (mm) & $57.6 \pm 8.08$ & $55.52 \pm 10.08$ \\
LVDs (mm) & $39.0 \pm 8.94$ & $40.65 \pm 8.71$ \\
IVS (mm) & $9.5 \pm 1.80$ & $9.31 \pm 1.92$ \\
LVPW (mm) & $9.6 \pm 1.86$ & $9.3 \pm 1.72$ \\
\hline
\end{tabular}

SR, sinus rhythm; AF, atrial fibrillation; NYHA, New York Heart Association classification; RVOT, right ventricular outflow tract; LAD, 1 eft atrial dimension; LVDd, 1eft ventricular internal dimension at end diastole; LVDs, 1eft ventricular internal dimension at end systole; IVS, interventricular septal thickness at end-diastole; LVPW, left ventricular posterior wal1. ${ }^{\mathrm{a}} \mathrm{p}<0.05$ with respect to SR patients.

\section{Results}

Patient characteristics. There were no significant differences in terms of age or New York Heart Association (NYHA) classifications between the SR and AF groups. Moreover, pre-operative Color Doppler echocardiography showed no differences in RVOT, LVDd, LVDs, IVS and LVPW, with the exception of LAD (Table I), in line with a recent report that the left atrial size was significantly greater in patients with $\mathrm{AF}$ than in those with SR (11).

2-DE profiling of differentially expressed proteins between $S R$ and AF patients. To examine changes in protein expression in patients with AF, the proteome of atrial appendages was compared between patients with SR and those with AF using 2-DE with a broad $\mathrm{pH}$ gradient ( $\mathrm{pH}$ 3-10 non-linear). To avoid misidentifications due to gel-to-gel variations, only protein features that were present in at least $75 \%$ of the gels belonging to a given group (SR or AF) were considered in the analysis. A pair of representative 2-DE maps is shown in Fig. 1. After automatic spot detection, background subtraction and volume normalization, $802 \pm 47$ protein spots in the SR and $813 \pm 38$ protein spots in AF groups were detected. As a result, 30 protein spots exhibited $>2$-fold changes $(\mathrm{p}<0.05)$, 19 of which were successfully identified by MS (Fig. 1). Thirteen of the proteins identified were up-regulated in the AF gels, whereas 6 were up-regulated in the SR samples. All the detected proteins showed a difference of not more than $\sim 10 \mathrm{kDa}$ compared to the theoretical values calculated on the basis of the ORFs in the genome.

Identification of differentially expressed proteins. Of the 30 spots selected, proteins in 19 spots were positively identified (Table II). The average value of the MOWSE scores was 209, while the number of unique peptides identified by MS/ MS was 5. A representative MS map of spot \#3 is shown in Fig. 2A. A MS/MS map of the parent ions (m/z 814.9725) 
is shown in Fig. 2B, indicating that this peptide sequence is ITSAYLQDIENAYK, part of the sequence of the protein NADH dehydrogenase [ubiquinone] $1 \alpha$ subcomplex subunit 10, mitochondrial (NDUAA). As shown in Fig. 2C and D, the output of the database search by MASCOT resulted in the identification of NDUAA.

Functional classification. Functional classification of the identified proteins was performed using the Gene Ontology annotation system. A total of 19 proteins were revealed to be differentially expressed between the AF and SR groups. These proteins have functions in diverse biological processes, including energy metabolism, apoptosis and oxidative stress (Table III). Using cluster analysis (by Cluster 3.0 and Treeview), the proteins identified were divided into three major groups: cytoskeleton and myofilement associated proteins, proteins involved in energy metabolism and proteins associated with oxidative stress (Table III).

\section{Discussion}

AF the most common arrhythmia in clinical practice and results in cardiovascular morbidity and mortality. However, despite significant advances in our understanding of the mechanisms associated with AF, no effective biomarkers or viable therapeutic targets have been identified. In the present study, 2-DE-based proteomic analysis of human atrial tissue was carried out to identify novel biomarkers for diagnosis and new targets for therapy. Differentially expressed proteins in tissues from patients with AF versus matched controls with SR were compared, and 19 proteins were found to be differentially regulated between the groups. These can be divided

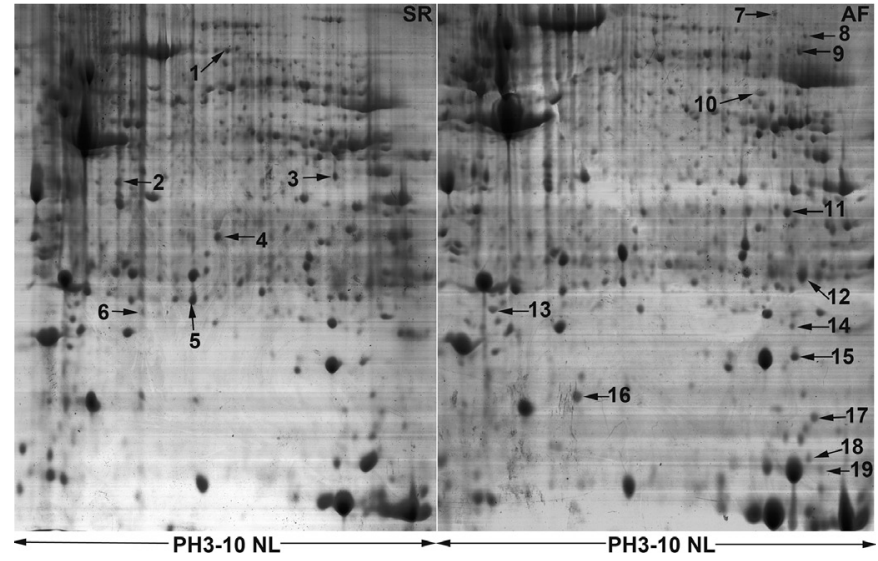

Figure 1. Representative 2-DE gel images corresponding to atrial tissue proteins from patients with AF and SR. Total protein extracts were separated on $\mathrm{pH}$ 3-10 non-linear IPG strips in the first dimension, followed by $12 \%$ SDS-PAGE in the second dimension, and visualized by Coomassie Brilliant Blue staining. In total, 19 differentially expressed spots were identified by MS/MS analysis (indicated by arrow and number). Data on each numbered spot is listed in Table II.

into three major groups: proteins involved in cytoskeleton and myofilament, energy metabolism associated proteins, and proteins associated with oxidative stress.

Cytoskeleton and myofilament. In AF patients, myosin light chain 3 (MLC3) and cardiac troponin I (cTnI) were up-regulated compared to the SR group. These play a critical role in regulating cardiac morphogenesis and construction. It is worth noting that, as members of the cardiac sarcomere proteins, mutations of these two genes have been associated with hyper-
A

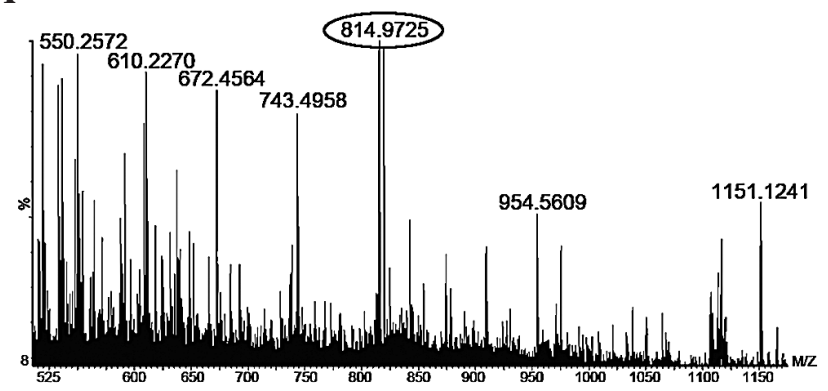

C

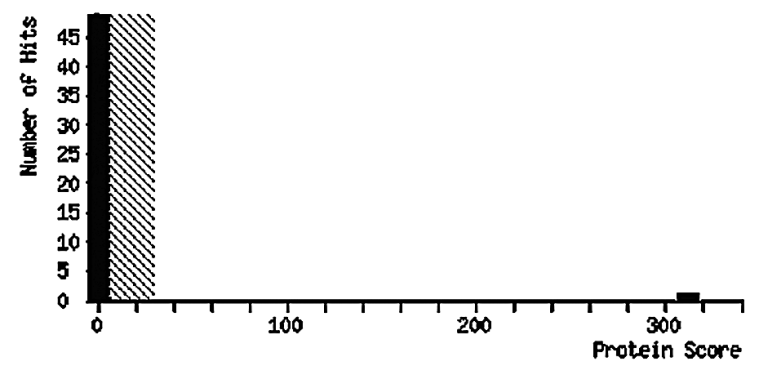

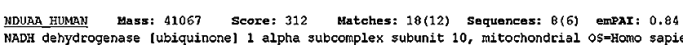

B

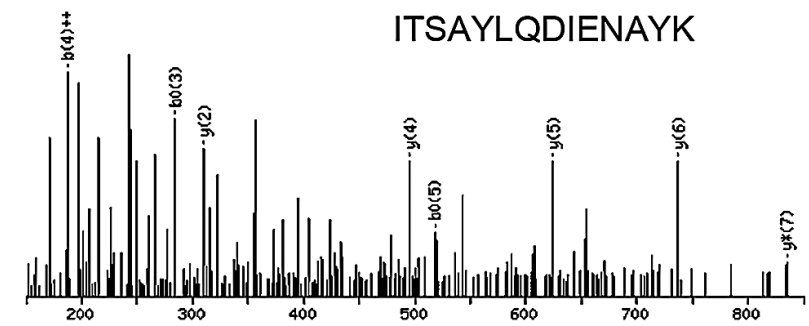

D

Figure 2. Identification of protein spot \#3. (A) Mass spectrogram of tryptic peptides from spot \#3. (B) An example of an MS/MS spectrum of parent ion 814.9725. (C) Output of a database search by MASCOT using MS/MS data, resulting in the identification of NDUAA. (D) Protein sequence of NDUAA. The matched peptides are underlined. 


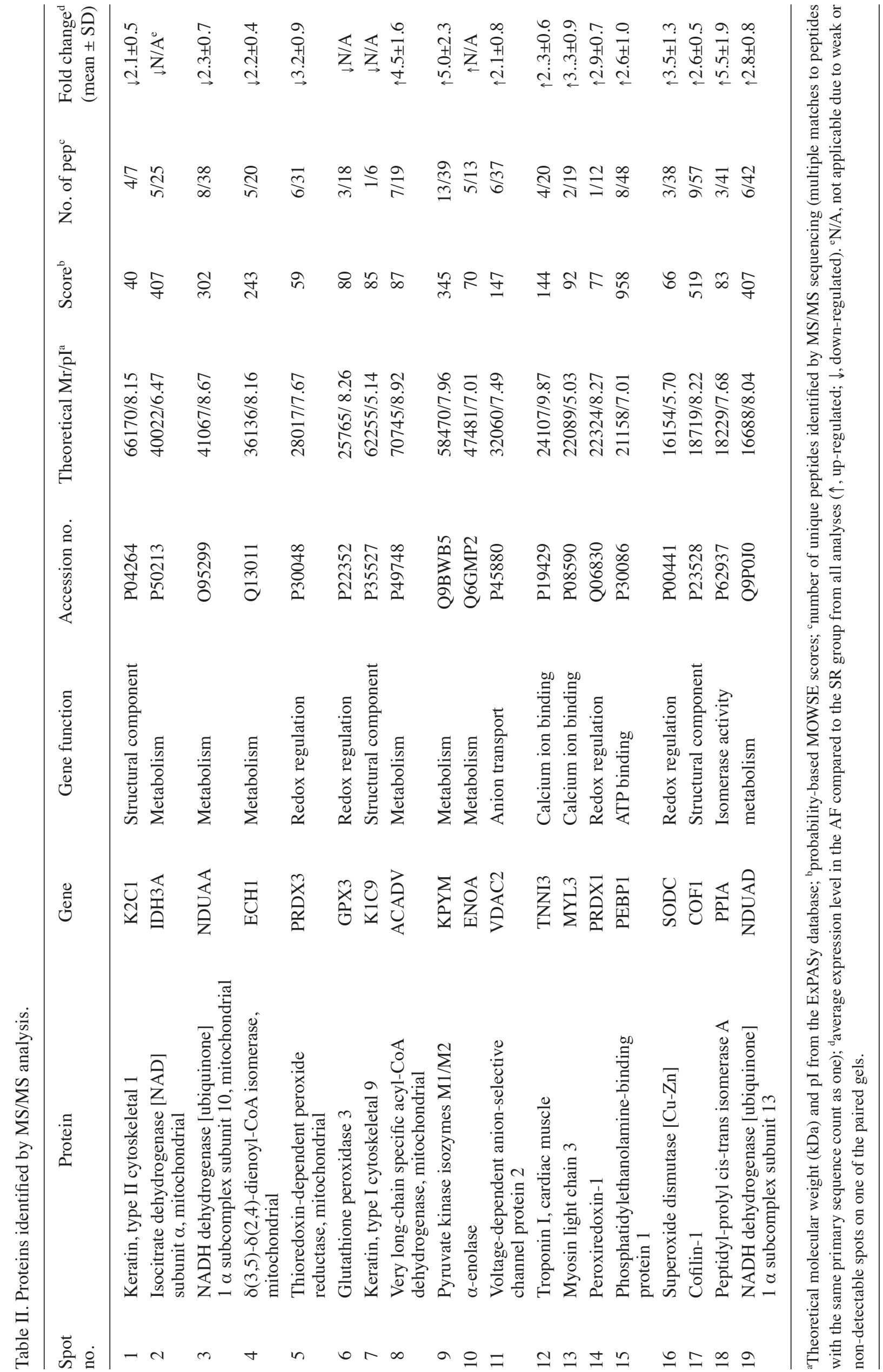


trophic cardiomyopathy (HCM) (15-17), while HCM patients have a greater likelihood of developing atrial fibrillation (18), which is also the most common arrhythmia observed in HCM (19). Combined with our results, these findings suggest that the mutation or deregulation of MLC3 and cTnI may be involved in the initiation and development of AF. Indeed, the downregulation of $\mathrm{cTnI}$ in the myocardium of patients with $\mathrm{AF}$ has been reported $(20,21)$, and this down-regulation has been found to be partly due to protein degradation by calpain (22). This seems to be in conflict with our present results. However, Thijssen et al (23) proposed that the expression of cTnI was closely related to the stage of AF, i.e., cTnI expression was up-regulated in chronic AF compared to earlier-stage AF, in part due to the suppression of degradation.

Both types of keratin, type II cytoskeletal 1 (K2C1) and type I cytoskeletal 9 (K1C9) keratin, are crucial structural constituents of the cytoskeleton. Notably, the expression of $\mathrm{K} 2 \mathrm{C} 1$ in the heart is the second highest in humans, as shown by the EST profile from Unigene. Although $\mathrm{K} 2 \mathrm{C} 1$ was downregulated and K1C9 was markedly up-regulated in AF with respect to SR patients, there have been no reports regarding the relationship between these two proteins and AF. Further studies are required to clarify the relationship between keratins and AF.

Energy metabolism. The maintenance of the normal function of the ion channels, such as calcium, sodium and potassium channel proteins, is critical for electric stability, which depends on sufficient energy supply. It has been shown that energy metabolism and arrhythmia are interdependent: the deregulated cellular energetic state predisposes patients to atrial arrhythmias, while atrial rhythm disturbances also influence metabolic activity (24). In this study, seven proteins involved in energy metabolism were differentialy expressed in human atrial tissues from AF and SR patients. These play essential roles in processes including fat acid $\beta$-oxidation, glycolysis, electron transport and the tricarboxylic acid cycle (TAC). Here, we mainly examined the identified proteins associated with fat acid $\beta$-oxidation and glycolysis.

Fat acid $\beta$-oxidation. Very long-chain specific acyl-CoA dehydrogenase, mitochondrial (ACADV) and $\delta(3,5)-\delta(2,4)$ dienoyl-CoA isomerase, mitochondria 1 (ECH1) all function in the fat acid $\beta$-oxidation pathway, which produces the main energy substrate-acetyl-CoA (25). ECH1 expression was down-regulated in AF, while ACADV was up-regulated. However, considering that, as opposed to the auxiliary effect of ECH1, ACADV catalyzes the initiated step of mitochondrial $\beta$-oxidation of straight-chain fatty acid, the fatty acid of $\beta$-oxidation appeared to be eventually enhanced in $\mathrm{AF}$ as a compensatory mechanism, since atrial fibrillation is a hypermetabolic state (26).

Glycolysis. Expression levels of the pyruvate kinase isozymes M1/M2 (KPYM) and $\alpha$-enolase (ENOA) were increased in samples from AF versus SR patients. Both catalyze the transfer of a phosphoryl group from phosphoenolpyruvate to ADP in the penultimate step of glycolysis, generating ATP and pyruvate. Increased expression of these two proteins may be a longer-term decreased energy saving adaptation in response to
Table III. Protein classification according to Gene Ontology.

\begin{tabular}{|c|c|}
\hline Groups & Proteins \\
\hline $\begin{array}{l}\text { Cytoskeleton and } \\
\text { myofilament }\end{array}$ & $\begin{array}{l}\text { Myosin light chain } 3 \text { (MYL3) } \\
\text { Troponin I, cardiac muscle (TNNI3) } \\
\text { Keratin, type II cytoskeletal } 1 \text { (K2C1) } \\
\text { Keratin, type I cytoskeletal } 9 \text { (K1C9) } \\
\text { Cofilin-1 (COF1) }\end{array}$ \\
\hline \multirow[t]{7}{*}{ Energy } & $\begin{array}{l}\text { Very long-chain specific metabolism } \\
\text { acyl-CoA dehydrogenase, } \\
\text { mitochondrial (ACADV) }\end{array}$ \\
\hline & $\begin{array}{l}\delta(3,5)-\delta(2,4) \text {-dienoyl-CoA isomerase, } \\
\text { mitochondrial }(\mathrm{ECH} 1)\end{array}$ \\
\hline & $\alpha$-enolase (ENOA) \\
\hline & $\begin{array}{l}\text { Pyruvate kinase isozymes } \\
\text { M1/M2 (KPYM) }\end{array}$ \\
\hline & $\begin{array}{l}\text { NADH dehydrogenase [ubiquinone] } \\
1 \alpha \text { subcomplex subunit } 10 \\
\text { mitochondrial (NDUAA) }\end{array}$ \\
\hline & $\begin{array}{l}\text { NADH dehydrogenase [ubiquinone] } \\
1 \alpha \text { subcomplex subunit } 13 \text { (NDUAD) }\end{array}$ \\
\hline & $\begin{array}{l}\text { Isocitrate dehydrogenase [NAD] } \\
\text { subunit } \alpha \text {, mitochondrial (IDH3A) }\end{array}$ \\
\hline \multirow{3}{*}{$\begin{array}{l}\text { Oxidative } \\
\text { stress }\end{array}$} & Peroxiredoxin-1 (PRDX1) \\
\hline & $\begin{array}{l}\text { Thioredoxin-dependent peroxide } \\
\text { reductase, mitochondrial (PRDX3) } \\
\text { Superoxide dismutase } \\
\text { [Cu-Zn] (SODC) }\end{array}$ \\
\hline & Glutathione peroxidase 3 (GPX3) \\
\hline \multirow[t]{3}{*}{ Other } & $\begin{array}{l}\text { Peptidyl-prolyl cis-trans } \\
\text { isomerase A (PPIA) }\end{array}$ \\
\hline & $\begin{array}{l}\text { Phosphatidylethanolamine-binding } \\
\text { protein } 1 \text { (PEBP1) }\end{array}$ \\
\hline & $\begin{array}{l}\text { Voltage-dependent anion-selective } \\
\text { channel protein } 2 \text { (VDAC2) }\end{array}$ \\
\hline
\end{tabular}

increased metabolic needs. Other enzymes involved in different energy metabolism pathways, such as electron transport and TAC, were differentially expressed in atrial appendages from $\mathrm{AF}$ and SR patients, which further suggests that there is a close relationship between energy metabolism and AF.

Oxidative stress. Accumulating evidence has demonstrated the involvement of oxidative stress in atrial tissue during AF, suggesting it plays a role in the remodeling phenomenon (27-29). Furthermore, several pharmacological approaches with non-channel-blocking drugs with antioxidant and antiinflammatory properties have shown beneficial effects on AF development (30-33).

Peroxiredoxin-1 (PRDX1) and thioredoxin-dependent peroxide reductase, mitochondrial (PRDX3) are members of the peroxiredoxin family of antioxidant enzymes, and play an antioxidant protective role in cells. Within erythrocytes, PRDX1 protected hemoglobin from irreparable damage inflicted by reactive oxygen species, which otherwise leads to anemia $(34,35)$. Additionally, it suppressed tyrosine kinase signaling, potentially regulating vascular remodeling (36). 
Combined with the up-regulation of PRDX1 in AF patients observed in this study, it is possible that PRDX1 protects the heart from oxidative damage during AF. PRDX3 confers protection against oxidative stress within the mitochondria of aortic cells and within the ischemic rat myocardium $(37,38)$. The decrease in the expression of PRDX3 in our AF sample is in line with previous observations that PRDX3 is downregulated in the failing myocardium, at least in terms of mRNA expression (39).

Superoxide dismutase [Cu-Zn] (SOD3) is another antioxidant enzyme that catalyzes the dismutation of two superoxide radicals into hydrogen peroxide and oxygen, and is thought to protect the brain, lungs, heart and other tissues from oxidative stress. SOD3 deficiency has been found to exacerbate transverse aortic constriction-induced myocardial oxidative injury (40). Overexpression of SOD3 protected against myocardial infarction-induced congestive heart failure (41). We speculated that the increased expression of SOD3 observed in AF versus SR patients has a protective effect in patients with AF.

Study limitations. In the present study, only a small number of patients were investigated due to the difficulty of finding RHD patients with SR. Although the left atrium plays a key role in the development of AF, only right atrial appendage tissues could be obtained during cardiac surgery. However, a recent study concluded that there are similarities between the right and left atrial appendage, at least in terms of the expression levels of proteins associated with the cytoskeleton, energy metabolism and cardiac cytoprotection (11). Another limitation was that the biological effects of the identified proteins were not validated in a biological model, since an appropriate mammalian cell model of AF has yet to be developed. Further studies using animal models of AF to explore the biological effects of the identified differently expressed proteins will soon be conducted by our group.

In conclusion, the data presented in this study may enable a better understanding of the molecular mechanisms of AF and may aid in drug development.

\section{Acknowledgements}

This study was supported by a grant from the National Natural Science Foundation of China (no. 30890903). The authors sincerely thank Zhi-Chao Zhou from the Erasmus Medical Center (Rotterdam) for help with the language.

\section{References}

1. Nattel S: New ideas about atrial fibrillation 50 years on. Nature 415: 219-226, 2002.

2. Kannel WB and Benjamin EJ: Status of the epidemiology of atrial fibrillation. Med Clin North Am 92: 17-40, 2008.

3. Lloyd-Jones DM, Wang TJ, Leip EP, et al: Lifetime risk for development of atrial fibrillation: the Framingham Heart Study Circulation 110: 1042-1046, 2004.

4. Heeringa J, van der Kuip DA, Hofman A, et al: Prevalence, incidence and lifetime risk of atrial fibrillation: the Rotterdam study. Eur Heart J 8: 949-953, 2006.

5. Go AS, Hylek EM, Phillips KA, Chang Y, Henault LE, Selby JV and Singer DE: Prevalence of diagnosed atrial fibrillation in adults: national implications for rhythm management and stroke prevention: the Anticoagulation and Risk Factors in Atrial Fibrillation (ATRIA) Study. JAMA 285: 2370-2375, 2001.
6. Miyasaka Y, Barnes ME, Gersh BJ, et al: Secular trends in incidence of atrial fibrillation in Olmsted County, Minnesota, 1980 to 2000, and implications on the projections for future prevalence. Circulation 114: 119-225, 2006.

7. Barderas MG, Dardé VM, Durán MC, Egido J and Vivanco F: Characterization of circulating human monocytes by proteomic analysis. Methods Mol Biol 357: 319-328, 2007.

8. Lai LP, Lin JL, Lin CS, et al: Functional genomic study on atrial fibrillation using cDNA microarray and two-dimensional protein electrophoresis techniques and identification of the myosin regulatory light chain isoform reprogramming in atrial fibrillation. $\mathrm{J}$ Cardiovasc Electrophysiol 15: 214-223, 2004.

9. De Souza AI, Cardin S, Wait R, et al: Proteomic and metabolomic analysis of atrial profibrillatory remodelling in congestive heart failure. J Mol Cell Cardiol 49: 853-861, 2010.

10. Mayr M, Yusuf S, Weir G, et al: Combined metabolomic and proteomic analysis of human atrial fibrillation. J Am Coll Cardiol 51: 585-594, 2008.

11. Modrego J, Maroto L, Tamargo J, et al: Comparative expression of proteins in left and right atrial appendages from patients with mitral valve disease at sinus rhythm and atrial fibrillation. J Cardiovasc Electrophysiol 21: 859-868, 2010.

12. García A, Eiras S, Parguina AF, et al: Gonzalez-Juanatey, Highresolution two-dimensional gel electrophoresis analysis of atrial tissue proteome reveals down-regulation of fibulin-1 in atrial fibrillation. Int J Cardiol: May, 2010 (E-pub ahead of print).

13. Tong A, Zhang H, Li Z, et al: Proteomic analysis of liver cancer cells treated with suberonylanilide hydroxamic acid. Cancer Chemother Pharmacol 61: 791-802, 2008.

14. Tong A, Wu L, Lin Q, et al: Proteomic analysis of cellular protein alterations using a hepatitis B virus-producing cellular model. Proteomics 8: 2012-2023, 2008.

15. Poetter K, Jiang H, Hassanzadeh S, et al: Epstein, mutations in either the essential or regulatory light chains of myosin are associated with a rare myopathy in human heart and skeletal muscle. Nat Genet 13: 63-69, 1996.

16. Andersen PS, Havndrup O, Bundgaard H, et al: Myosin light chain mutations in familial hypertrophic cardiomyopathy: phenotypic presentation and frequency in Danish and South African populations. J Med Genet 38: E43, 2001.

17. Richard RP, Charron P, Carrier L, et al: Hypertrophic cardiomyopathy: distribution of disease genes, spectrum of mutations, and implications for a molecular diagnosis strategy. Circulation 107: 2227-2232, 2003.

18. Maron BJ, Casey SA, Poliac LC, Gohman TE, Almquist AK and Aeppli DM: When compared with the general population, HCM patients have greater likelihood of developing atrial fibrillation. JAMA 281: 650-655, 1999.

19. Olivotto I, Cecchi F, Casey SA, Dolara A, Traverse JH and Maron BJ: Impact of atrial fibrillation on the clinical course of hypertrophic cardiomyopathy. Circulation 104: 2517-2524, 2001.

20. Thijssen VL, van der Velden HM, van Ankeren EP, et al: Analysis of altered gene expression during sustained atrial fibrillation in the goat. Cardiovasc Re 54: 427-437, 2002.

21. Ke L, Qi XY, Dijkhuis AJ, et al: Calpain mediates cardiac troponin degradation and contractile dysfunction in atrial fibrillation. J Mol Cell Cardiol 45: 685-693, 2008.

22. Brundel BJ, Ausma J, van Gelder IC, van der Wan JJ, van Gilst WH, Crijns HJ and Henning RH: Activation of proteolysis by calpains and structural changes in human paroxysmal and persistent atrial fibrillation. Cardiovasc Res 54: 380-389, 2002.

23. Thijssen VL, Ausma J, Gorza L, et al: troponin I isoform expression in human and experimental atrial fibrillation. Circulation 110: 770-775, 2004.

24. Barth AS and Tomaselli GF: Cardiac metabolism and arrhythmias. Arrhythm Electrophysiol 2: 327-335, 2009.

25. Taegtmeyer H: Energy metabolism of the heart: from basic concepts to clinical applications. Curr Probl Cardiol 19: 59-113, 1994.

26. Barth AS, Merk S, Arnoldi E, et al: Reprogramming of the human atrial transcriptome in permanent atrial fibrillation: expression of a ventricular-like genomic signature. Circ Res 96: 1022-1029, 2005.

27. Korantzopoulos P, Kolettis T, Siogas K and Goudevenos J: Atrial fibrillation and electrical remodeling: the potential role of inflammation and oxidative stress. Med Sci Monit 9: 225-229, 2003.

28. Van Wagoner DR: Molecular basis of atrial fibrillation: a dream or a reality. J Cardiovasc Electrophysiol 14: 667-669, 2003.

29. Van Wagoner DR: Electrophysiological remodeling in human atrial fibrillation. Pacing Clin Electrophysiol 26: 1572-1575, 2003. 
30. Shinagawa K, Derakhchan K and Nattel S: Pharmacological prevention of atrial tachycardia induced atrial remodeling as a potential therapeutic strategy. Pacing Clin Electrophysiol 26: $752-764,2003$

31. Goette A and Lendeckel U: Nonchannel drug targets in atrial fibrillation. Pharmacol Ther 102: 17-36, 2004.

32. Leonardi M and Bissett J: Prevention of atrial fibrillation. Curr Opin Cardiol 20: 417-423, 2005

33. Lozano HF, Conde CA, Florin T and Lamas GA: Treatment and prevention of atrial fibrillation with nonantiarrhythmic pharmacologic therapy. Heart Rhythm 2: 1000-1007, 2005.

34. Lee TH, Kim SU, Yu SL, et al: Peroxiredoxin II is essential for sustaining life span of erythrocytes in mice. Blood 101: 5033-5038, 2003.

35. Neumann CA, Krause DS, Carman CV, et al: Essential role for the peroxiredoxin Prdx1 in erythrocyte antioxidant defence and tumour suppression. Nature 424: 561-565, 2003.

36. Schroder E and Eaton P: Hydrogen peroxide as an endogenous mediator and exogenous tool in cardiovascular research: issues and considerations. Curr Opin Pharmacol 8: 153-159, 2008.
37. Araki M, Nanri H, Ejima K, Murasato $Y$, Fujiwara $T$, Nakashima $\mathrm{Y}$ and Ikeda M: Antioxidant function of the mitochondrial protein SP-22 in the cardiovascular system. J Biol Chem 274: 2271-2278, 1999.

38. Matsushima S, Ide T, Yamato M, et al: Overexpression of mitochondrial peroxiredoxin-3 prevents left ventricular remodeling and failure after myocardial infarctionin mice. Circulation 113: 1779-1786, 2006.

39. Brixius K, Schwinger RH, Hoyer F, et al: Isoform-specific downregulation of peroxiredoxin in human failing myocardium Life Sci 81: 823-831, 2007.

40. Lu Z, Xu X, Hu X, et al: Extracellular superoxide dismutase deficiency exacerbates pressure overload-induced left ventricular hypertrophy and dysfunction. Hypertension 51: 19-25, 2008.

41. Van Deel ED, Lu Z, Xu X, Zhu G, Hu X and Oury TD: Extracellular SOD protects the heart against oxidative stress and hypertrophy after myocardial infarction. Free Radic Biol Med 44: 1305-1313, 2008 